\title{
QCD evolution based evidence for the onset of gluon saturation in exclusive photo-production of vector mesons
}

\author{
Martin Hentschinski ${ }^{* \dagger}$ \\ Departamento de Actuaria, Física y Matemáticas Universidad de las Américas Puebla \\ Ex-Hacienda Santa Catarina Martir S/N, San Andrés Cholula 72820 Puebla, Mexico \\ E-mail: martin.hentschinski@udlap.mx
}

\begin{abstract}
We investigate photo-production of vector mesons J/Psi and Upsilon measured both at HERA and LHC, using 2 particular fits of inclusive unintegrated gluon distributions. We find that linear next-to-leading order evolution can only describe production at highest energies, if perturbative corrections are increased to unnaturally large values; rendering this corrections to a perturbative size, the growth with energy is too strong and the description fails. At the same time, the KS gluon, which we explore both with and without non-linear corrections, requires the latter to achieve an accurate description of the energy dependence of data. We interpret this observation as a clear signal for the presence of high gluon densities in the proton, characteristic for the onset of gluon saturation.
\end{abstract}

European Physical Society Conference on High Energy Physics - EPS-HEP2019 -

10-17 July, 2019

Ghent, Belgium

\footnotetext{
* Speaker.

${ }^{\dagger}$ Collaboration with Alfredo Arroyo Garcia and Krzysztof Kutak as well as support by Consejo Naciona de Ciencia y Tecnología (Ciencias Básica SEP-CONACyt) project number A1-S-43940 is gratefully acknowledged.
} 


\section{Introduction}

The observed power-like rise of the gluon distribution at small $x$, with $x=M^{2} / s$ the ratio of the hard scale $M$ and the center of mass energy $\sqrt{s}$ cannot continue forever. Unitarity dictates that the observed rise in $x$ must eventually slow down and come to hold. This is to be achieved through the formation of an over occupied system of gluons, which eventually leads to saturation of gluon densities [1]; finding convincing phenomenological evidence for gluon saturation is one of the open problems of Quantum Chromodynamics (QCD). The evolution from the low to large gluon densities is described by a set of nonlinear evolution equations, known as Balitsky-JalilianMarian-Iancu-McLerran-Weigert-Leonidov-Kovner; its frequently used mean field version is given by the Balitsky Kovchegov (BK) [2] evolution equation.

\section{Results}

At the Large Hadron Collider, the region of interest, namely very low $x$ and a hard scale close to the non-perturbative boundary, can be investigated by exclusive photo-production of vector mesons. For this observable a large amount of data has been collected both for the production of $J / \Psi$ and $\Upsilon$ vector mesons. The hard scale is in both cases provided by the heavy quark mass, i.e. the charm $(J / \Psi)$ and bottom ( $\Upsilon$ ) quark mass. In the case of the $J / \Psi$ one is therefore able to reach very small $x$ values at a low transverse scale, which allows for the potential observation of saturation effects. Photo-production of the $\Upsilon$ provides on the other hand a cross-check of the description well in the perturbative domain, where such effects are genuinely expected to be absent. In the following we present our results, comparing both linear next-to-leading order BFKL evolution, the Hentschinski-Salas-Sabio Vera (HSS)[7] unintegrated gluon and a particular solution to BKevolution, with initial conditions fitted to combined HERA data by Kutak-Sapeta (KS) [11]. At first we find that both linear and non-linear evolution describe data, see Fig. 1, if the hard scale is identified with the a typical external scale, such as the heavy quark mass. Taking however a closer look at the dipole cross-section associated with the HSS-gluon one realizes that the perturbative expansion underlying the particular fit turns out the be unstable for the highest LHC energies, see [9] for a detailed discussion. This instability is easily cured, if one fixes the hard scale instead to the inverse transverse separation of the quark-anti-quark dipole. Indeed both for $\Upsilon$ production and $J / \Psi$ production in the HERA region, changing the renormalization scale merely leads to a slight shift of the result, comparable to the order of a typical variation of the renormalization scale, see e.g. [10]. On the other hand the stabilized linear NLO BFKL evolution overshoots data for the $J / \Psi$ in the LHC region. At the same time the energy dependence of the data is very well described by the non-linear KS gluon. To assess the importance of the non-linearities in the solution, we also compare to the KS-gluon with non-linearities turned off (dashed black line): We observe that the linear KS-gluon overshoots data. We therefore conclude that non-linear effects are essential to describe the energy dependence of $J / \Psi$ data in the LHC region. We interpret this as a clear sign for the onset of saturation effects in this region of phase space.

\section{Conclusions}

The observed slow-down of the growth with energy is one of the core predictions of gluon 

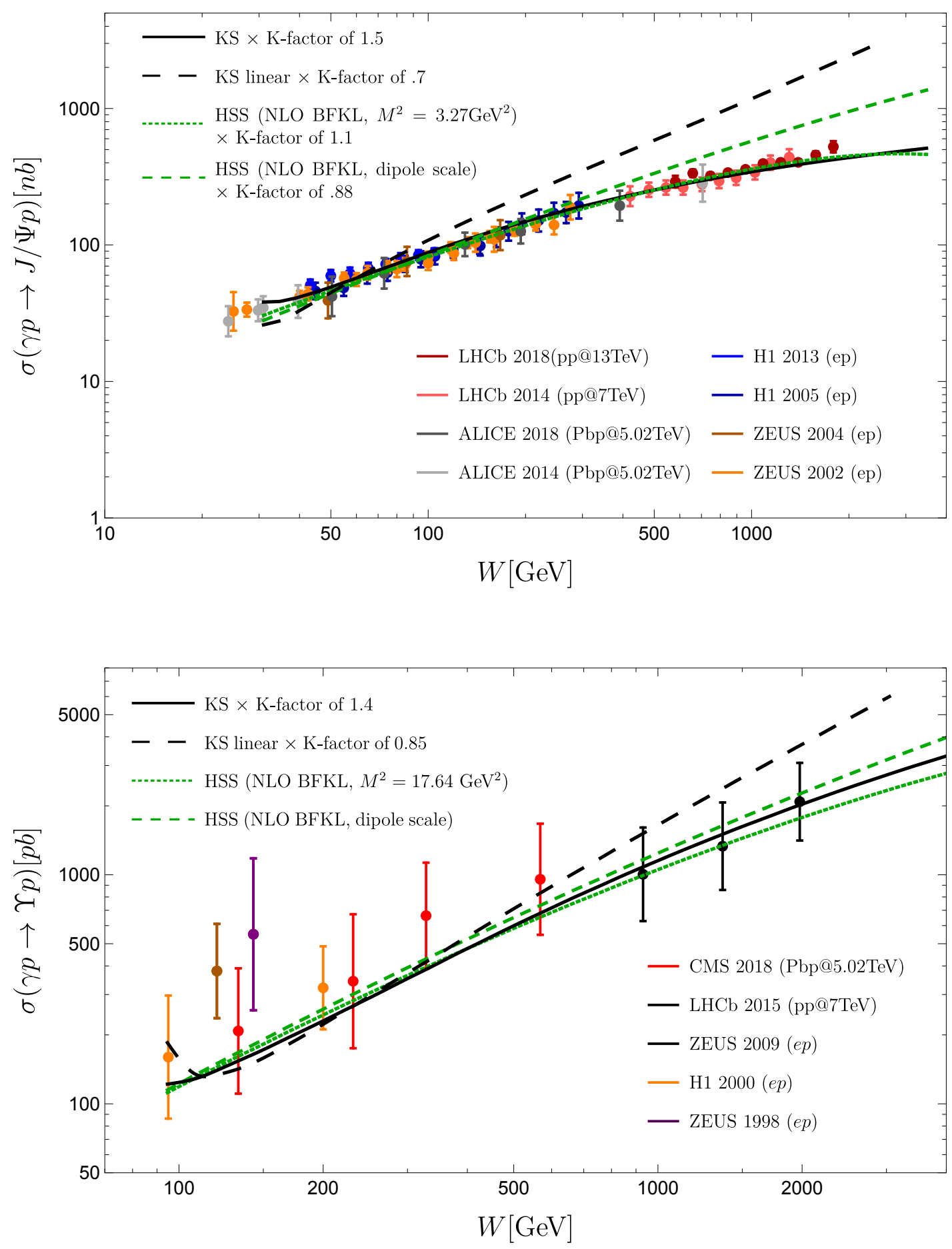

Figure 1: Energy dependence of the $J / \Psi$ and $\Upsilon$ photo-production cross-section as provided by the KS and HSS gluon distribution. The HSS distribution with dipole size scale corresponds to a specific scale setting for the HSS gluon discussed in Sec. 2. For the $J / \Psi$ we further display photo-production data measured at HERA by ZEUS and HI collaborations [3] as well as LHC data obtained from ALICE and LHCb [4]. For the $\Upsilon$ cross-section we show HERA data measured by HI and ZEUS [5] and LHC data by LHCb and CMS $[6]$. 
saturation, which we find in data, once we ensure stability of the NLO BFKL evolution. It is worth to note in this context that [12] arrives at a similar conclusion for the process of photo-production of vector mesons. To further establish the observation made in this contribution it is necessary to search for different observables which probe the low $x$ gluon in a similar kinematic regime and to increase further the theoretical accuracy of the underlying framework.

\section{References}

[1] L. V. Gribov, E. M. Levin and M. G. Ryskin, Phys. Rept. 100 (1983) 1. doi:10.1016/0370-1573(83)90022-4

[2] I. Balitsky, Nucl. Phys. B 463 (1996) 99 doi:10.1016/0550-3213(95)00638-9 [hep-ph/9509348]; Y. V. Kovchegov, Phys. Rev. D 60 (1999) 034008 doi:10.1103/PhysRevD.60.034008 [hep-ph/9901281].

[3] S. Chekanov et al. [ZEUS Collaboration], Eur. Phys. J. C 24, 345 (2002) [hep-ex/0201043];

S. Chekanov et al. [ZEUS Collaboration], Nucl. Phys. B 695, 3 (2004) [hep-ex/0404008]; C. Alexa et al. [H1 Collaboration], Eur. Phys. J. C 73, no. 6, 2466 (2013) [arXiv:1304.5162 [hep-ex]]; A. Aktas et al. [H1 Collaboration], Eur. Phys. J. C 46, 585 (2006) [hep-ex/0510016].

[4] B. B. Abelev et al. [ALICE Collaboration], Phys. Rev. Lett. 113, no. 23, 232504 (2014) [arXiv:1406.7819 [nucl-ex]]; S. Acharya et al. [ALICE Collaboration], Eur. Phys. J. C 79, no. 5, 402 (2019) doi:10.1140/epjc/s10052-019-6816-2 [arXiv:1809.03235 [nucl-ex]];

R. Aaij et al. [LHCb Collaboration], J. Phys. G 40, 045001 (2013) [arXiv:1301.7084 [hep-ex]]; J. Phys. G 41, 055002 (2014) [arXiv:1401.3288 [hep-ex]]; R. Aaij et al. [LHCb Collaboration], JHEP 1810, 167 (2018) doi:10.1007/JHEP10(2018)167 [arXiv:1806.04079 [hep-ex]].

[5] C. Adloff et al. [H1 Collaboration], Phys. Lett. B 483, 23 (2000) [hep-ex/0003020]; J. Breitweg et al. [ZEUS Collaboration], Phys. Lett. B 437 (1998) 432 [hep-ex/9807020]; S. Chekanov et al. [ZEUS Collaboration], Phys. Lett. B 680, 4 (2009) [arXiv:0903.4205 [hep-ex]].

[6] R. Aaij et al. [LHCb Collaboration], JHEP 1509, 084 (2015) [arXiv:1505.08139 [hep-ex]]; CMS Collaboration [CMS Collaboration], "Measurement of exclusive $\mathrm{Y}$ photoproduction in $\mathrm{pPb}$ collisions at $\sqrt{s_{\mathrm{NN}}}=5.02 \mathrm{TeV}$," CMS-PAS-FSQ-13-009; A. M. Sirunyan et al. [CMS Collaboration], Eur. Phys. J. C 79, no. 3, 277 (2019) doi:10.1140/epjc/s10052-019-6774-8 [arXiv:1809.11080 [hep-ex]].

[7] M. Hentschinski, A. Sabio Vera and C. Salas, Phys. Rev. D 87, no. 7, 076005 (2013) doi:10.1103/PhysRevD.87.076005 [arXiv:1301.5283 [hep-ph]]; Phys. Rev. Lett. 110 (2013) no.4, 041601 [arXiv:1209.1353 [hep-ph]].

[8] K. Kutak and S. Sapeta, Phys. Rev. D 86 (2012) 094043 doi:10.1103/PhysRevD.86.094043 [arXiv:1205.5035 [hep-ph]].

[9] A. Arroyo Garcia, M. Hentschinski and K. Kutak, Phys. Lett. B 795, 569 (2019) doi:10.1016/j.physletb.2019.06.061 [arXiv:1904.04394 [hep-ph]].

[10] I. Bautista, A. Fernandez Tellez and M. Hentschinski, Phys. Rev. D 94, no. 5, 054002 (2016) doi:10.1103/PhysRevD.94.054002 [arXiv:1607.05203 [hep-ph]].

[11] K. Kutak and S. Sapeta, Phys. Rev. D 86 (2012) 094043 doi:10.1103/PhysRevD.86.094043 [arXiv:1205.5035 [hep-ph]].

[12] D. Schildknecht, Phys. Lett. B 769 (2017) 166 doi:10.1016/j.physletb.2017.03.056 [arXiv:1611.01382 [hep-ph]]. 